\title{
Study on Morphological Properties of Polyvinyl Alcohol/Poly(lactic acid) Wound Dressing Membrane as Drug Delivery Carrier in Wound Healing Treatment
}

\author{
Celine Ng${ }^{1}$, Mohd Syahir Anwar Hamzah ${ }^{1}$, Saiful Izwan Abd Razak ${ }^{2}$, Jumadi \\ Abdul Sukor ${ }^{1}$, Nafarizal Nayan ${ }^{3,4}$, Nadirul Hasraf Mat Nayan ${ }^{1,4 *}$
}

${ }^{1}$ Faculty of Engineering Technology,

Universiti Tun Hussein Onn Malaysia, Pagoh Higher Education Hub, Jalan Panchor, Pagoh, 84600, MALAYSIA

${ }^{2}$ Centre of Advanced Composite, Faculty of Engineering,

Universiti Teknologi Malaysia, Jalan Universiti, Skudai, 81310, MALAYSIA

${ }^{3}$ Faculty of Electrical and Electronics

Universiti Tun Hussein Onn Malaysia, Parit Raja, 86400, MALAYSIA

${ }^{4}$ Microelectronics \& Nanotechnology-Shamsudin Research Centre,

Universiti Tun Hussein Onn Malaysia, Parit Raja, 86400, MALAYSIA

*Corresponding Author

DOI: https://doi.org/10.30880/jaita.2020.01.02.002

Received 22 June 2020; Accepted 09 September 2020; Available online 15 December 2020

\begin{abstract}
Wound dressing have experienced continuous improvement and changes since ancient time. Electrospinning of polymeric nanofibers have captured the interest of researchers due to its simplicity and cost effective technique that able to produce wound dressing membrane that meet the requirement as ideal wound dressing and drug delivery carrier. In this work, polyvinyl alcohol (PVA) and poly (lactic acid) (PLA) were chosen polymer to produce wound dressing membrane through electrospinning and coating method. PVA was electrospun and then coated with PLA. Preliminary study had been conducted between 1, 4 and $8 \%$ concentration for the selection of optimum PLA coating concentration. PLA has been proposed for the use of coating materials at $4 \%$, the coated nanofiber membrane started to exhibit high Ultimate Tensile Strength at $1022.5 \pm 9.28 \mathrm{MPa}$, highest degree of swelling $(1850 \pm 3.7 \%)$ and optimum water contact angle $\left(60.9 \pm 11.46^{\circ}\right)$. The aim of this study was to investigate the morphological properties of PVA/PLA wound dressing membrane. Based on the results from Atomic Force Microscope, PVA nanofiber coated with 4\% PLA exhibit the highest value of Rq which is $0.47 \pm$ $0.19 \mu \mathrm{m}$ compared to neat PVA nanofibers membrane. Field Emission Scanning Electron Microscope (FE-SEM) image revealed that PVA nanofiber coated with 4\% PLA shows porous fiber-like morphology and well incorporated with each other without any gap formed between them. This report clearly suggestive of the fact that synthetic biodegradable polymers such as PLA can be exploited for the synergistic combination with PVA nanofiber for wound dressing application.
\end{abstract}

Keywords: Wound, electrospinning, polyvinyl alcohol, poly (lactic acid), morphological

\section{Introduction}

Electrospinning derived from the term "electrostatic spinning" is a simple and effective fabrication technique that has gain attention in the last twenty years due to its versatility and ability to consistently produced micro- and nano- 
fibers with high surface-to-volume ratio [1]. Advantages such as high surface-to-volume ratio, high porosity and enhanced physico-mechanical properties offered by nanofibers made it broadly applied in biomedical application such as tissue engineering scaffold, drug delivery system and wound dressing [2].

An ideal wound dressing should have haemostatic ability, efficiency as bacterial barrier, absorption ability of excess fluid, appropriate water vapour transmission rate, adequate gaseous exchange ability, ability to conform to the contour of the wound area, functional adhesion and ease of removal [1]. Electrospun materials meet most of the requirement outlined for the development of ideal wound dressing for wound healing [3]. Figure 1 illustrate the functions provided by electrospun nanofibers as ideal wound dressing.

In this study, polyvinyl alcohol (PVA) and poly (lactic acid) (PLA) were the selected as the fabrication candidate of wound dressing membrane through electrospinning and coating method. PVA is a hydrophilic synthetic polymer, which able to absorb large amount of water without dissolving. However, in some application, PVA exhibit low mechanical strength which may lead to inefficient of drug release. Excessive drug release can cause side effect to human body [4, 5]. In terms of controlled drug release due to its hydrophobic characteristic, a study proven that incorporation of PLA enhanced the hydrophobicity of chitosan blend for controlled drug release [6]. Due to this reason, PLA was proposed not only used to increase the mechanical strength of PVA nanofiber but also act as a protective layer to control drug release. Up to date, there is no previous studies that investigate the morphology of PVA electrospun nanofibers coated with PLA. Hence, the objective of this work was to investigate the effect of PLA coating on morphological properties of PVA nanofibers through Atomic Force Microscope (AFM) and Field Emission Scanning Electron Microscope (FE-SEM).

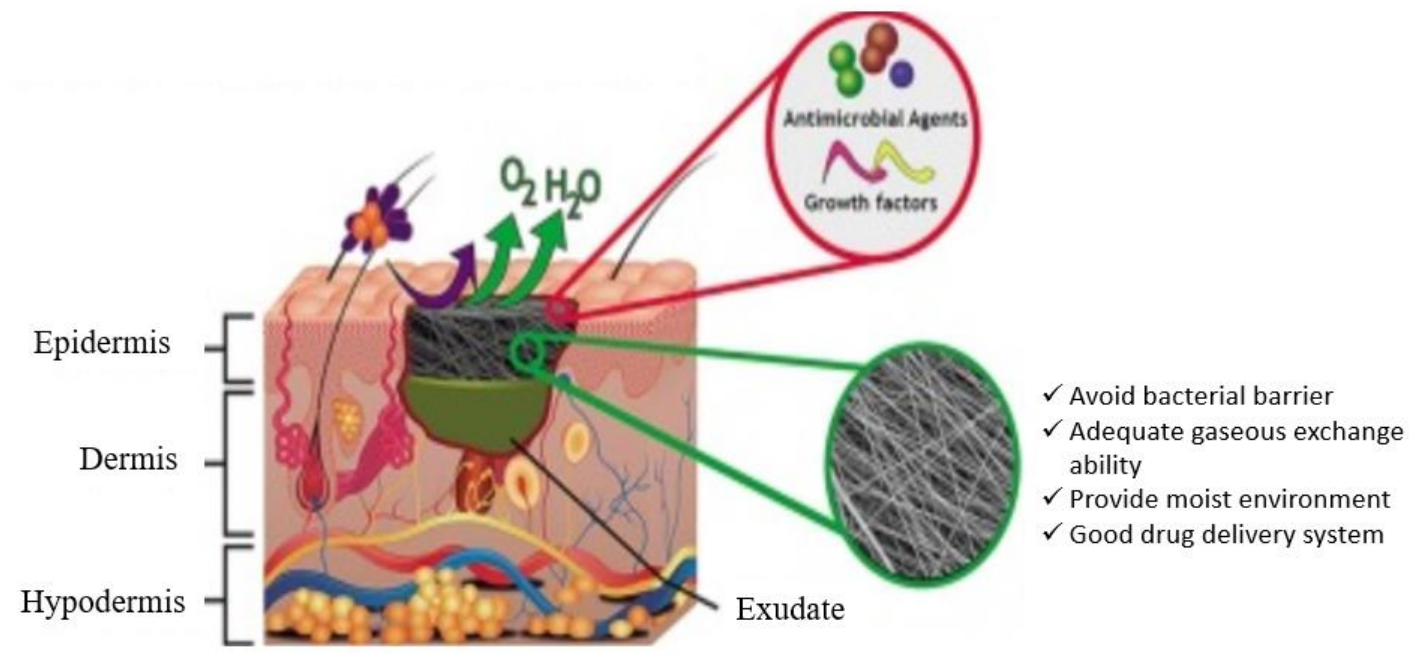

Fig. 1 - Illustration of electrospun nanofibers as ideal wound dressing [1,3]

\section{Methodology}

This section described the techniques used for sample preparation and sample testing. The PVA/PLA wound dressing membrane preparation was explained in first part of this section and followed by the second part which is the sample testing method and instrument.

\subsection{PVA/PLA Wound Dressing Membrane Sample Preparation}

$0.5 \mathrm{~g}$ of polyvinyl alcohol (PVA) powder (Mw $\approx 89000 \mathrm{~g} / \mathrm{mol}$, Sigma Aldrich) was dissolved in $5 \mathrm{~mL}$ of distilled water to form $10 \%(\mathrm{w} / \mathrm{v})$ solution of PVA. An electrospinning device with horizontal set up (eSpinner NF-COEN/II, Birooni Saintifik) was used for the nanofabrication of PVA. The electrospinning condition for PVA sample were: flow rate $=1.5 \mathrm{~mL} / \mathrm{h}$, voltage $=15 \mathrm{kV}$, gauge needle $=0.7 \mathrm{~mm}$ and the tip-to-collector distance $=10 \mathrm{~cm}$. Poly $($ lactic acid $)$ (PLA) granules (Mw $\approx 60000 \mathrm{~g} / \mathrm{mol}$, Sigma Aldrich) are dissolved in organic solvent that contained dichloromethane (Sigma Aldrich) and N,N-Dimethyl formamide (Sigma Aldrich) in a ratio of 3:2 to produce three different coating concentration: $1 \%, 4 \%$ and $8 \%(\mathrm{w} / \mathrm{v})$. PVA nanofiber is then immersed in PLA solution for 15 minutes and dried in fume hood by allowing the evaporation of the organic solvent under ambient temperature [7]. Figure 2 shows the process of sample preparation. 


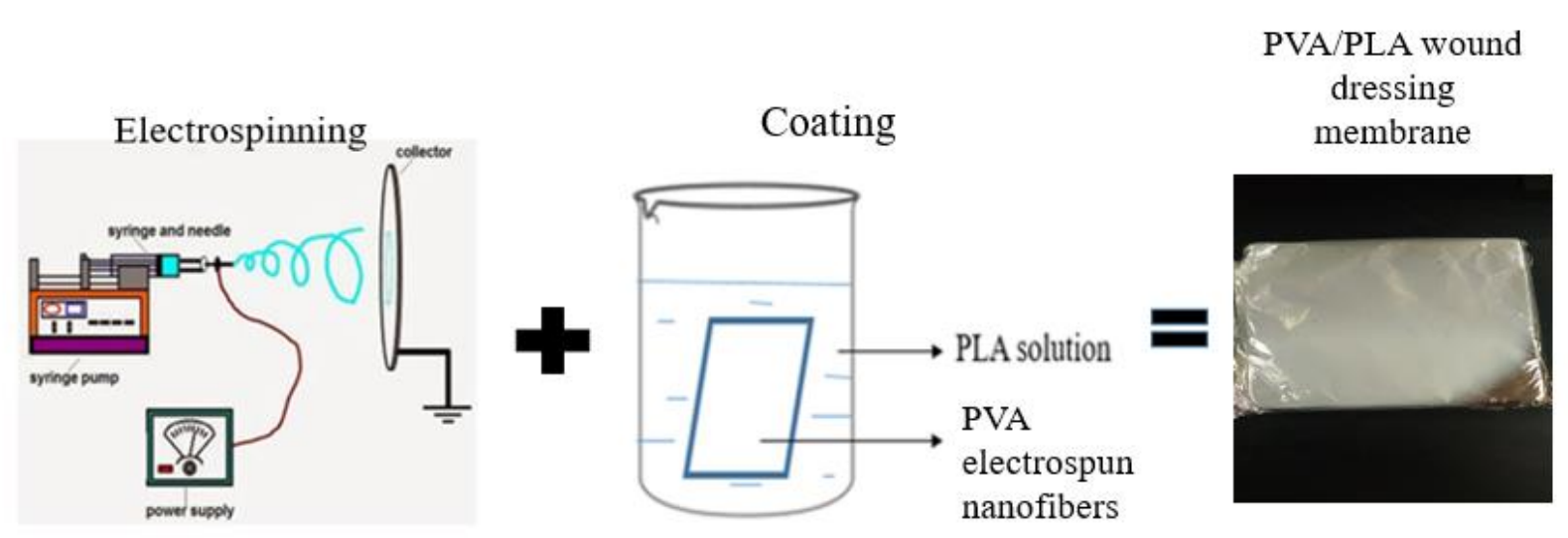

Fig. 2 - Preparation of polyvinyl alcohol nanofiber mat coated with poly(lactic acid)

\subsection{Morphological Properties Characterization}

\section{Atomic Force Microscope}

Atomic Force Microscope uses a cantilever tip to image a sample and is capable to provide extremely high resolution image. The sample was prepared into $1 \mathrm{~cm} \times 1 \mathrm{~cm}$ (length $\mathrm{x}$ width). The surface topology of nanofiber mat was observed by AFM (Innova Ris, Bruker).

\section{Scanning Electron Microscope}

Scanning Electron Microscope (SEM) (FlexSEM 1000, Hitachi) was used for morphological observation of polyvinyl alcohol nanofiber mat coated with poly(lactic acid). The nanofiber mat was sputtered coated with gold for 30 $\mathrm{s}$ at $18 \mathrm{~mA}$ and being examined with $1000 \mathrm{X}-10000 \mathrm{X}$ magnification.
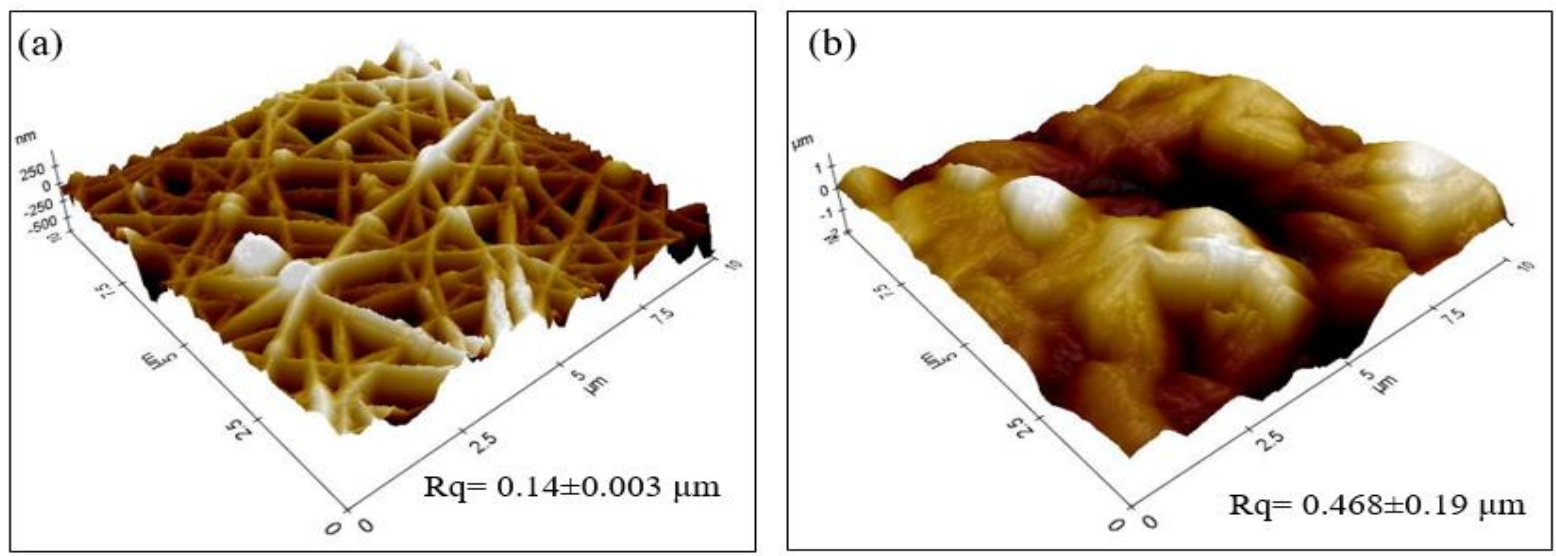

Fig. 3 - AFM images for (a) neat PVA, (b) PVA electrospun nanofiber coated with $4 \%$ PLA

\section{Results and Discussion}

\section{Atomic Force Microscope}

Preliminary study has been conducted to select the optimum PLA coating concentration on PVA electrospun nanofibers. Between 1\%, 4\% and 8\% (w/v) PLA, 4\% PLA shows the most promising result of mechanical properties and physical properties through tensile test, degree of swelling and water contact angle. The coated nanofiber mat started to exhibit high Ultimate Tensile Strength (1022.5 \pm 9.28 MPa), high Young Modulus (257.56 $\pm 11.37 \mathrm{MPa})$, high degree of swelling $(1850 \pm 3.7 \%)$ and optimum water contact angle $\left(60.9 \pm 11.46^{\circ}\right)$. Thus, for the morphological properties investigation, only neat PVA electrospun nanofibers and 4\% PLA coated PVA electrospun nanofibers samples were tested and analyzed. Atomic Force Microscopy (AFM) is an imaging tool used to characterize the surface roughness of a material. Figure 3 (a) and (b) shows the AFM images for neat PVA, bilayer PVA/4\%PLA wound dressing membrane respectively. Figure 3 (a) revealed the image of the neat PVA nanofibers mat. As for Figure 3 (b), it 
shows uneven surface and this was due to the coating of 4\% PLA on the PVA nanofibers. The higher the Rq value, the rougher the surface [8]. Based on the figures revealed, the coated nanofiber exhibit the highest value of Rq which is $0.47 \pm 0.19 \mu \mathrm{m}$. The rougher the surface, the higher the water contact angle which indicate higher wetting time.

\section{Field Emission Scanning Electron Microscope (FE-SEM)}

Figure 4 (a) shows the FE-SEM image of electrospun neat PVA nanofiber. The micrograph of the electrospun nanofiber mat shows the presence of nanofiber threads at 35000x magnification. The image revealed that there are beads formation on the nanofibers. The possible reason for the formation of the beads could be the flow rate setting during electrospinning process. Previous studies shown that higher flow rate caused beads formation and the nanofiber formed were not so smooth [9]. The average diameter of the neat PVA nanofiber is $143.57 \pm 18.07 \mathrm{~nm}$. The coating of 4\% PLA on bilayer PVA nanofiber can be seen in Figure 4 (b). It has more fibrous morphology where maximum amount of drug can be loaded and being released in a moderate rate which is essential for wound healing [10]. Moreover, the coating of PLA filled up the empty spaces on the surface of bilayer PVA nanofiber which improved the low mechanical strength of PVA. Figure 4 (c) shows the cross-section image of bilayer PVA nanofiber coated with $4 \%$ PLA. From the figure, it can be seen that PLA incorporate well with the nanofiber where there is no gap between them. From the morphological results obtained, it shows that PLA layer could be a good candidate of biomaterial in biomedical application such as wound dressing and drug delivery system.
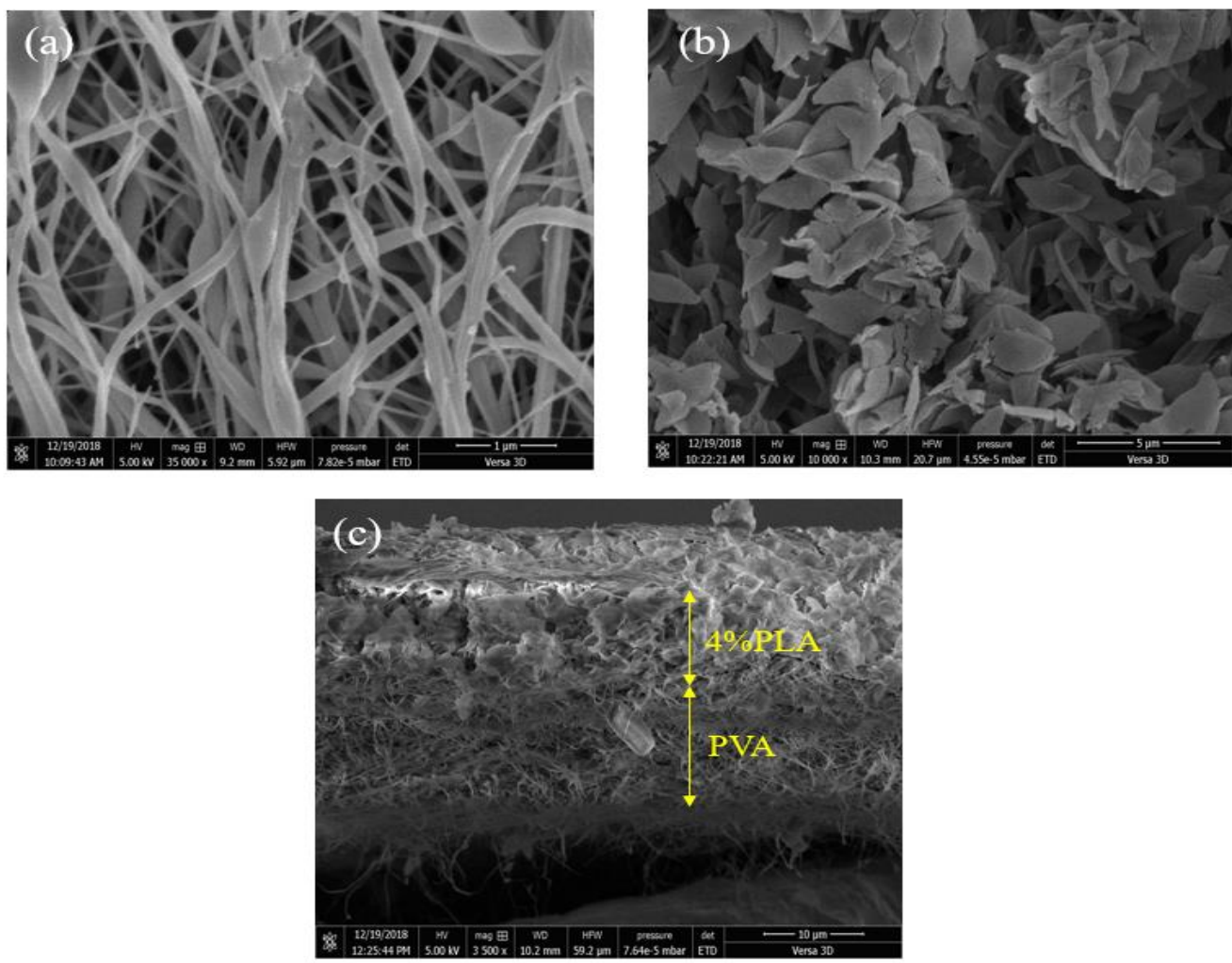

Fig. 4 - FE-SEM image of (a) neat PVA nanofiber, (b) PVA nanofiber coated with 4\% PLA and (c) cross-section of PVA nanofiber coated with $4 \%$ PLA

\section{Conclusion}

In this study, the purpose of coating is to strengthen the mechanical properties and to provide a protective layer on surface of nanofiber in order to control drug release rate in wound dressing. By conducting morphological properties testing and analyzation, PVA/4\% PLA wound dressing membrane showed rougher surface and porous-fibrous like morphology. Well-incorporation of PVA and PLA proved that these polymers have high potential to be used as biomaterial in biomedical application such as drug delivery carrier and wound dressing membrane. Other than that, coating of PLA layer on PVA nanofibers membrane revealed promising morphological results which capable to act as protective layer to control the release of drug to wound site efficiently. 


\section{Acknowledgement}

The authors would like to express their deepest appreciation to the Universiti Tun Hussein Onn Malaysia (UTHM) through Geran Penyelidikan Pascasiswazah (GPPS-Vote number H599) and Industrial Grant (Vote number M009) as well as Ministry of Education Malaysia (MOE) through the Fundamental Research Grant Scheme (FRGS-Vote number K220), Malaysia Technical University Network Grant Scheme (MTUN-Vote number K124 and K243) for funding this research. The authors would also like to express their appreciation to Universiti Teknologi Malaysia (UTM) for the use of laboratory for conducting the research.

\section{References}

[1] Bhardwaj, N. \& Kundu, S.C. (2010). Electrospinning: A Fascinating Fiber Fabrication Technique. Biotechnology Advances, 28 (3), 325-347

[2] Jayarama, Reddy, V., Radhakrishnan, S., Ravichandran, R., Mukherjee, S., Balamurugan, R., Sundarrajan, S. \& Ramakrishna, S. (2013). Nanofibrous Structured Biomimetic Strategies for Skin Tissue Regeneration. Wound and Repair Regeneration, 21(1), 1-16

[3] Najafabadi, A.H., Tamayol, A., Annabi, N., Ochoa, M., Mostafalu, P., Akbari, M., Nikkhah, M., Rahimi, R., Dokmeci, M.R., Sonkusale, S., Ziaie, B. \& Khademhosseini, A. (2014). Biodegradable Nanofibrous Polymeric Substrates for Generating Elastic and Flexible Electronics. Advanced Materials. 26, 5823-5830

[4] Yang, J.M., Su, W.Y., Leu, T.L. \& Yang, M.C. (2004). Evaluation of Chitosan/PVA Blended Hydrogel Membranes. Journal of Membrane Science. 236, 39-51

[5] Gaaz, T.S., Sulong, A.B., Akhtar, M.N., Kadhum, A.A.H., Mohamad, A.B. \& Al-Amiery, A.A. (2015). Properties and Applications of Polyvinyl Alcohol, Halloysite Nanotubes and Their Nanocomposites. Molecules, 20, 2283322847

[6] Suyatma, N.E., Copinet, A., Tighzert, L. \& Coma, V. (2004). Mechanical and Barrier Properties of Biodegradable Films Made from Chitosan and Poly (Lactic Acid) Blends. Journal of Polymers and the Environment, 12(1), 1-6

[7] Choi, Y.F., Hamzah, M.S.A., Abd Razak, S.I., Saidin, S. \& Mat Nayan, N.H. (2017). Influence of Poly(lactic acid) Layer on the Physical and Antibacterial Properties of Dry Bacterial Cellulose Sheet for Potential Acute Wound Healing Materials. Fibers and Polymers, 19(2), 263-271

[8] Bajpai A.K., Bhatt R.K. \& Katare, R. (2016). Atomic Force Microscopy Enabled Roughness Analysis of Nanostructured Poly (diaminonaphthalene) Doped Poly (vinyl alcohol) Conducting Polymer Thin Films. Micron, 90, 12-17

[9] Zuo, W.W., Zhu, M.F., Yang, W., Yu, H., Chen, Y.M. \& Zhang, Y. (2005). Experimental Study on Relationship between Jet Instability and Formation of Beaded Fibers during Electrospinning. Polymer Engineering Science, 45, 704-709

[10] Zahedi, P., Rezaeian, I., Ranaei-Siadat, S.O., Jafari, S.H. \& Supaphol, P. (2009). A Review on Wound Dressings with an Emphasis on Electrospun Nanofibrous Polymeric Bandages. Polymers Advanced Technologies, 21, 7795 